\title{
Is there a future for informatics research? A strategy for the 21 st century
}

A Cranfield conference in honour of Jack Meadows June 28th and 29th 1999

\section{List of delegates}

\begin{tabular}{|c|c|c|}
\hline Mr Mayur Amin & $\begin{array}{l}\text { Assoc. Director Market } \\
\text { Research }\end{array}$ & Elsevier Science Ltd. \\
\hline Mr Nigel Atkinson & $\begin{array}{l}\text { UK Sales and Customer } \\
\text { Relations Director }\end{array}$ & Cambridge University Press \\
\hline Mr Toby Bainton & Secretary & SCONUL \\
\hline Mr Derek Barlow & Consultant & Cura Consortium \\
\hline Professor Micheline Beaulieu & $\begin{array}{l}\text { Head of Department of } \\
\text { Information Studies }\end{array}$ & Sheffield University \\
\hline Mr John Blagden & Former University Librarian & Cranfield University Librarian \\
\hline Professor Christine Borgman & $\begin{array}{l}\text { Professor and Presidential } \\
\text { Chair in Information Studies }\end{array}$ & $\begin{array}{l}\text { University of California at Los } \\
\text { Angeles }\end{array}$ \\
\hline Mr Roger Bowes & Chief Executive & ASLIB/IMI \\
\hline Mr David Brown & Business Development Director & Ingenta Ltd. \\
\hline Mr Peter Burnhill & Director & EDINA \\
\hline Mr Robert Campbell & Managing Director & Blackwell Science Ltd. \\
\hline Mr Tony Cawkell & Director & Citech Ltd. \\
\hline Mr Neil Charlesworth & Executive Chairman & The Charlesworth Group \\
\hline Professor Mel Collier & $\begin{array}{l}\text { Director of Strategic and } \\
\text { Operational Planning }\end{array}$ & Dawson Holdings PLC \\
\hline Dr Bernard Dixon & Consultant & Former Editor New Scientist \\
\hline Mrs Diana Dixon & $\begin{array}{l}\text { Hon. Sec. LA Information } \\
\text { Services Group }\end{array}$ & \\
\hline Professor Bernard Donovan & Former Secretary General & $\begin{array}{l}\text { Association of Learned and } \\
\text { Professional Society Publishers } \\
\text { (ALPSP) }\end{array}$ \\
\hline Dr Harry East & Senior Research Fellow & City University \\
\hline Mr Mark Furneaux & MD European Operations & Cambridge Scientific Abstracts \\
\hline Professor Eugene Garfield & Chairman Emeritus & Inst. for Scientific Information \\
\hline Mr. Alan Gilchrist & $\begin{array}{l}\text { Editor, Journal of Information } \\
\text { Science }\end{array}$ & Cura Consortium \\
\hline Dr Mark Goodwin & Editor & Open University Publishing \\
\hline
\end{tabular}




\begin{tabular}{|c|c|c|}
\hline Ms Margaret Haines & Chief Executive & $\begin{array}{l}\text { Library and Information } \\
\text { Commission }\end{array}$ \\
\hline Ms Linda Hajdukiewicz & Commissioning Editor & Bowker-Saur \\
\hline Mr Tim Hamer & Managing Director & ISI Europe \\
\hline Professor Frank Hartley & Vice-Chancellor & Cranfield University \\
\hline Ms Helen Henderson & Consultant & The Gallery \\
\hline Ms Ros Herman & Freelance Journalist & Sciencewords \\
\hline Mr Michael Holdsworth & $\begin{array}{l}\text { Press Business Development } \\
\text { Manager }\end{array}$ & Cambridge University Press \\
\hline Mr Simon Inger & Managing director & Catchword Ltd. \\
\hline Dr Donald King & $\begin{array}{l}\text { Researcher, Former President } \\
\text { King Research }\end{array}$ & University of Michigan \\
\hline Mr Derek Law & $\begin{array}{l}\text { Librarian and Director of } \\
\text { Information Strategy }\end{array}$ & University Strathclyde \\
\hline Dr Peter Leggate & Keeper of Scientific Books & $\begin{array}{l}\text { Radcliffe Science Library } \\
\text { Oxford University }\end{array}$ \\
\hline Mr Chris Leamy & Consultant & The Gallery \\
\hline Professor Yves F. Le Coadic & $\begin{array}{l}\text { Information Science } \\
\text { Professor }\end{array}$ & $\begin{array}{l}\text { Conservatoire National des } \\
\text { Arts Memers }\end{array}$ \\
\hline Professor Maurice Line & $\begin{array}{l}\text { Information and Library } \\
\text { Consultant }\end{array}$ & \\
\hline Mr Barry Mahon & Consultant & $\begin{array}{l}\text { Former Executive Director } \\
\text { of Eusidic }\end{array}$ \\
\hline Dr Nigel Macartney & $\begin{array}{l}\text { Director Educational } \\
\text { Services }\end{array}$ & University of Ulster \\
\hline Professor Alan MacDougal & $\begin{array}{l}\text { Director of Information } \\
\text { Services and Systems }\end{array}$ & Kings College London \\
\hline Professor Cliff McKnight & $\begin{array}{l}\text { Head of Department of } \\
\text { Information Science }\end{array}$ & Loughborough University \\
\hline Professor Jack Meadows & $\begin{array}{l}\text { Information Science } \\
\text { Department }\end{array}$ & Loughborough University \\
\hline Mr Ian Middleton & General Manager & $\begin{array}{l}\text { EBSCO Information } \\
\text { Services }\end{array}$ \\
\hline Mrs Sally Morris & Secretary General & $\begin{array}{l}\text { Association of Learned and } \\
\text { Professional Society } \\
\text { Publishers (ALPSP) }\end{array}$ \\
\hline Professor Charles Oppenheim & $\begin{array}{l}\text { Professor of Information } \\
\text { Science }\end{array}$ & Loughborough University \\
\hline Ms Gillian Page & Consultant & Publishing \\
\hline Mr Albert Prior & Publisher Relations Manager & Swets and Zeitlinger \\
\hline Dr David Pullinger & Consultant & \\
\hline Ms Santa Rivelli & Marketing Manager & $I S I$ \\
\hline Dr Stephen Roberts & Senior Lecturer & Thames Valley University \\
\hline Professor Stephen Robertson & Researcher & Microsoft Research Ltd. \\
\hline Mr Dave Russon & Deputy Chief Executive & British Library \\
\hline
\end{tabular}




\begin{tabular}{|c|c|c|}
\hline Mr Fytton Rowland & $\begin{array}{l}\text { Director of Undergraduate } \\
\text { Programmes }\end{array}$ & $\begin{array}{l}\text { Information Science } \\
\text { Department Loughborough } \\
\text { University }\end{array}$ \\
\hline Professor Brian Shackel & Professor Emeritus-retired & $\begin{array}{l}\text { HUSAT Research Institute } \\
\text { Loughborough University }\end{array}$ \\
\hline Mr John Sumsion, O.B.E & Senior Honorary Fellow & $\begin{array}{l}\text { Information Science } \\
\text { Department }\end{array}$ \\
\hline Mr Stephen Town & Deputy University Librarian & Cranfield University - RMCS \\
\hline Mr Rollo Turner & Secretary & $\begin{array}{l}\text { Association of Subscription } \\
\text { Agents }\end{array}$ \\
\hline Dr Sandra Ward & Director & TFPL Ltd. \\
\hline Mr David Whitaker & Retired & \\
\hline Mrs Suzanne Wilson-Higgins & Marketing Director & $\begin{array}{l}\text { Blackwells Information } \\
\text { Services }\end{array}$ \\
\hline Sir Arnold Wolfendale, FRS & Emeritus Professor & University of Durham \\
\hline Dr Hazel Woodward & $\begin{array}{l}\text { University Librarian and } \\
\text { Director Cranfield Press }\end{array}$ & Cranfield University \\
\hline
\end{tabular}

\section{Dinner guests}

\begin{tabular}{|c|c|c|}
\hline Professor William Brock & $\begin{array}{l}\text { Emeritus Professor of } \\
\text { History and Science }\end{array}$ & Leicester University \\
\hline Dr Heather Couper & Creative Director & Pioneer TV Productions \\
\hline Mr Nigel Henbest & Director of Development & Pioneer TV Productions \\
\hline \multicolumn{3}{|l|}{ Mrs Val Hartley } \\
\hline Professor Phil Hutchinson & $\begin{array}{l}\text { Head of School of } \\
\text { Mechanical Engineering }\end{array}$ & Cranfield University \\
\hline Professor Raymond Hide & $\begin{array}{l}\text { Emeritus Professor of } \\
\text { Physics }\end{array}$ & University of Oxford \\
\hline \multicolumn{3}{|l|}{ Dr Joan Meadows } \\
\hline Dr John Roche & Linacre College & University of Oxford \\
\hline Prof. Peter and Mrs Joan Sneath & $\begin{array}{l}\text { Emeritus Prof. of } \\
\text { MicroBiology }\end{array}$ & Leicester University \\
\hline
\end{tabular}

\section{Sponsors}

We are grateful to the following organisations for their support of this Conference:
Aslib/imi
Blackwells Information Services
Blackwell Science
Bowker Saur
Cambridge Scientific Abstracts
Cambridge University Press
Catchword
Dawson UK Ltd.
Ebsco Information Services

\author{
Elsevier Science Ltd. \\ ingenta \\ ISI Institute for Scientific Information \\ OCLC Europe \\ Sirsi Ltd. \\ Swets Subscription Service \\ Taylor and Francis \\ The Charlesworth Group
}

